\title{
A third derivative test for mean values of exponential sums with application to lattice point problems
}

\author{
by \\ O. Robert and P. Sargos (Nancy)
}

\section{Introduction and statement of the result}

1.1. Third derivative test for exponential sums. Let $M$ be a large integer (say $M \geq 10$ ) and $\lambda$ be a small positive number (say $0<\lambda<1 / 10$ ). Let $f:[1, M] \rightarrow \mathbb{R}$ be a $C^{3}$ function which satisfies van der Corput's hypothesis:

$$
\lambda \leq f^{\prime \prime \prime}(x) \ll \lambda \quad \text { for } 1 \leq x \leq M,
$$

where the Vinogradov symbol $u \ll v$ means that $v$ is positive and $|u| \leq C v$ for some positive absolute constant $C$.

The exponential sum $S_{M}=\sum_{m=1}^{M} e(f(m))$, where we have set $e(x)=$ $e^{2 i \pi x}$, can be bounded by means of van der Corput's classical "third derivative test" (cf. [1, Theorem 2.6]):

$$
S_{M} \ll M \lambda^{1 / 6}+M^{3 / 4}+M^{1 / 4} \lambda^{-1 / 4} .
$$

For "short" exponential sums (say $M \ll \lambda^{-2 / 3}$ ), this bound has been sharpened to

$$
S_{M} \ll M \lambda^{1 / 6}+\lambda^{-1 / 3},
$$

independently by Gritsenko [3] and the second author [8]. But the exponent $1 / 6$ in (1.2) has never been improved, even for "long" exponential sums (say $\left.M \gg \lambda^{-1}\right)$. In fact, the second author has conjectured [9] that the bound

$$
S_{M} \ll M \lambda^{\vartheta},
$$

whenever $f$ satisfies (1.1), under the restriction $M \gg \lambda^{-1}$, does not hold for any $\vartheta>1 / 6$.

1.2. Third derivative test for the distribution of fractional parts. Now, we consider the analogous problem in terms of fractional parts. For any

2000 Mathematics Subject Classification: Primary 11L07. 
real $x$, we set $\psi(x)=\{x\}-1 / 2$. We introduce the sum

$$
\tau_{M}=\sum_{m=1}^{M} \psi(f(m)) .
$$

Giving upper bounds for $\tau_{M}$ is the key point in many lattice points problems (cf. [6], [1], [4]). For instance, under the assumption (1.1), one deduces from (1.3) that

$$
\tau_{M} \ll M \lambda^{1 / 7}+\lambda^{-1 / 3},
$$

and, without additional hypothesis, nothing more is known.

As above, the question whether the exponent $1 / 7$ can be increased (at least under some restrictions on the relative size of $M$ and $\lambda$ ) arises naturally. For comparison, in the same problem, but with the second derivative instead of the third one, the corresponding exponent is $1 / 3$ and a counter-example, due to Grekos [2], shows that, even in a much weaker problem, this last exponent can never be increased.

The aim of this paper is to show that the exponent $1 / 7$ can be increased to $3 / 19$. This is a consequence of a slightly more general problem that we describe now.

1.3. A third derivative test for mean values of exponential sums. Let $f, M, \lambda$ be as above. Let $H$ be a positive integer. We consider the following mean value of exponential sums:

$$
S=\frac{1}{H} \sum_{h=H+1}^{2 H}\left|\sum_{m=1}^{M_{h}} e\left(\frac{h}{H} f(m)\right)\right|,
$$

where the integers $M_{h}$ satisfy $1 \leq M_{h} \leq M$ for each $h \in[H+1,2 H]$.

A rough application of (1.3) yields the bound

$$
S \ll M \lambda^{1 / 6}+\lambda^{-1 / 3} .
$$

Our main result may be stated as follows:

THEOREM 1. With the above notations, and assuming (1.1), we have

$$
S \ll_{\varepsilon} M^{\varepsilon}\left(\frac{M \lambda^{1 / 6}}{H^{1 / 9}}+M \lambda^{1 / 5}+M^{3 / 4}\right)+\lambda^{-1 / 3} .
$$

Here and in what follows, the notation $\ll_{\varepsilon}$ means that the bound holds for each $\varepsilon>0$ and that the implied constant depends at most on $\varepsilon$ and on the previous implied constants.

The proof relies on the same mean value theorem for quadruple exponential sums as in [7]. But this one works much stronger here, because the phase function in (1.7) is now linear in $h$. In return, the optimization, with respect to the various parameters introduced in the proof, becomes intricate. 
From Theorem 1, we derive our main application in a standard way:

THEOREM 2. If we assume (1.1), then

$$
\sum_{m=1}^{M} \psi(f(m)) \ll_{\varepsilon} M^{1+\varepsilon} \lambda^{3 / 19}+M^{3 / 4+\varepsilon}+\lambda^{-1 / 3} .
$$

REMARK. The problem of giving upper bounds for $\sum_{m=1}^{M} \psi(f(m))$ is mostly studied with more hypotheses on $f$, namely under the hypotheses of the method of exponent pairs (cf. $[1, \S 3.3])$. All best results to date have been obtained by Huxley (cf. $[4, \S 18.5]$ ), combining deep methods stemming from Bombieri and Iwaniec's method, and are summed up in Tables 18.1 of $[4]$.

If we restrict our Theorem 2 to such functions, then our bound (1.10) is not entirely contained in Huxley's result: our bound is slightly better when $\alpha$ is close to 1.450 in Table 18.1 of [4].

Moreover, additional hypotheses on $f$ would yield stronger estimations in the "second spacing problem" with the corresponding improvement in Theorems 1 and 2. This will be done by the first author in a forthcoming paper.

Notations. We only use classical notations:

$u \ll v$ or $u=O(v)$ means that $u$ is a complex number, $v$ is a positive number and there exists an absolute constant $C$ which depends at most on previous constants such that $|u| \leq C v$;

$u \ll_{\varepsilon} v$ or $u=O_{\varepsilon}(v)$ means furthermore that the bound holds for each $\varepsilon>0$ and that the constant $C$ may depend on $\varepsilon$.

$u \asymp v$ means that both $u \ll v$ and $v \ll u$.

We denote by $\|x\|$ the distance of $x$ to the nearest integer.

The symbol - means that the proof is finished or has been omitted.

The function $\psi$ is defined on $\mathbb{R}$ by the formula $\psi(t)=\{t\}-1 / 2=$ $t-[t]-1 / 2$, where $[t]$ is the integer part of $t$, and $\{t\}$ is the fractional part of $t$.

2. Lemmas from the previous paper. Our method is quite similar to that of our previous paper [7]. We sum up the exact results we need by means of independent lemmas.

2.1. A Diophantine system. Let $R, Q, H, N$ be positive integers and $\delta$ be a positive number. We denote by $\mathcal{N}(R, Q, H, N ; \delta)$ the number of integer points $\left(r_{1}, r_{2}, q_{1}, q_{2}, h_{1}, h_{2}, n_{1}, n_{2}\right) \in \mathbb{Z}^{8}$ lying in the domain:

$$
\left\{\begin{array}{l}
0<\left|r_{i}\right| \leq R, \quad Q \leq\left|q_{i}\right|<2 Q, \quad H \leq h_{i}<2 H, \\
1 \leq n_{i} \leq N \quad \text { for } i=1,2, \quad q_{1} q_{2}>0
\end{array}\right.
$$


and satisfying the system of two equations and an inequality:

$$
\left\{\begin{array}{l}
r_{1}=r_{2}, \\
r_{1} n_{1}+h_{1} q_{1}=r_{2} n_{2}+h_{2} q_{2}, \\
\left|r_{1} n_{1}^{2}+2 h_{1} q_{1} n_{1}+h_{1} q_{1}^{2}-\left(r_{2} n_{2}^{2}+2 h_{2} q_{2} n_{2}+h_{2} q_{2}^{2}\right)\right| \leq \delta H Q^{2} .
\end{array}\right.
$$

LEMMA 1. If $R \leq H / 2$, then we have the bound

$$
\mathcal{N}(R, Q, H, N ; \delta) \ll_{\varepsilon}(R Q H N)^{1+\varepsilon}(1+\delta Q)
$$

This is Theorem 2 of [7].

2.2. The "second spacing lemma". According to Huxley's terminology [4], the double large sieve method for exponential sums uses two spacing lemmas. Here, the first one is Lemma 1, and the second one is the following.

Let $f:[1, M] \rightarrow \mathbb{R}$ be a $C^{3}$ function. We set $\mathcal{B}=\mathcal{B}(R, Q, H, N ; f)=$ the number of $\left(m_{1}, m_{2}\right) \in\{1, \ldots, M\}^{2}$ which satisfy the system of three inequalities:

$$
\left\{\begin{array}{l}
\left|f^{\prime \prime}\left(m_{1}\right)-f^{\prime \prime}\left(m_{2}\right)\right| \leq(Q N)^{-1} \\
\left\|\left(f^{\prime}\left(m_{1}\right)-f^{\prime}\left(m_{2}\right)\right) / H\right\| \leq(H Q)^{-1} \\
\left\|\left(f\left(m_{1}\right)-f\left(m_{2}\right)\right) / H\right\| \leq R^{-1}
\end{array}\right.
$$

Lemma 2. Suppose that the hypothesis (1.1) is satisfied. Then

$$
\mathcal{B} \ll M+\frac{M}{\lambda H Q^{2} N}+\frac{\log M}{Q \lambda} .
$$

The proof of (2.4) goes as in Step 6 in Section 4 of [7]. We note that this proof does not use the third inequality of (2.3). More hypotheses on $f$ would yield a better bound in (2.4) and thus would improve Theorems 1 and 2 .

2.3. The main inequality. Let $f:[1, M] \rightarrow \mathbb{R}$ be a $C^{3}$ function that satisfies (1.1) and let $H \geq 2$ be an integer. We want to bound $S$, where $S$ is defined in (1.7).

For this, we choose integer parameters $R, Q, N$ which satisfy

$$
1 \leq R \leq H / 2, \quad 1 \leq Q \ll N \ll \lambda^{-1 / 3}, \quad R N \ll H Q, \quad M Q \lambda \gg 1 .
$$

LEMMA 3. With the above hypotheses and notations we have the bound

$$
S \ll_{\varepsilon} M^{\varepsilon}\left(\frac{M}{(Q R)^{1 / 2}}+M(Q \lambda)^{1 / 4}\left(\frac{\mathcal{B}}{M}\right)^{1 / 4}+\left(\frac{M S_{1}}{Q}\right)^{1 / 2}+(M N)^{1 / 2}\right),
$$

where we have set $\mathcal{B}=\mathcal{B}(R, Q, H, N ; f)$ and

$$
S_{1}=\frac{1}{R} \sum_{r=1}^{R}\left|\sum_{m=1}^{M} e\left(\frac{r}{H} f(m)\right)\right| \text {. }
$$


Proof. (a) We want to bound

$$
S=\frac{1}{H} \sum_{h=H+1}^{2 H}\left|\sum_{m=1}^{M_{h}} e\left(\frac{h}{H} f(m)\right)\right| .
$$

But we may suppose that $M_{h}=M$ for each $h$ thanks to Lemma 5.2 .3 of [4] and we only have to pay a factor $\log M$. From now on, we suppose that $M_{h}=M$ for each $h$.

(b) We write

$$
S=\frac{1}{H} \sum_{h=H+1}^{2 H} a(h) \sum_{m=1}^{M} e\left(\frac{h}{H} f(m)\right)
$$

where $a(h)$ is some complex number of modulus 1 , and we apply van der Corput's lemma $A \times A$ (cf. [7, Lemma 1]) to the above double sum to get

$$
S \ll \frac{M}{(Q R)^{1 / 2}}+\left(\frac{M S_{1}}{Q}\right)^{1 / 2}+\left(\frac{M S_{2}}{R}\right)^{1 / 2}+\left(\frac{M S_{3}}{H Q R}\right)^{1 / 2}+M^{1 / 2} Q^{1 / 2}
$$

where we have set

$$
\begin{aligned}
& S_{2}=\frac{1}{Q H} \sum_{q \neq 0} \sum_{h}\left|\sum_{m} e\left(\frac{h}{H}(f(m+q)-f(m))\right)\right|, \\
& S_{3}=\left|\sum_{0<|r| \leq R} \sum_{0<|q| \leq Q} \sum_{h=H+1}^{2 H} \sum_{m=Q+1}^{M-Q} b(r, q, h) e\left(\frac{h}{H} f(m+q)-\frac{h+r}{H} f(m)\right)\right|
\end{aligned}
$$

and where $S_{1}$ is defined as in (2.7). The coefficients $b(r, q, h)$ are complex numbers of modulus at most one; the term $O\left(M^{1 / 2} Q^{1 / 2}\right)$ in the above inequality comes from the $O(Q)$ values of $m$ which are not counted in $S_{3}$.

We apply van der Corput's inequality (cf. [1, Theorem 2.2]) to bound the exponential sum in $S_{2}$ and we get

$$
S_{2} \ll M(Q \lambda)^{1 / 2}+(Q \lambda)^{-1 / 2} \ll M(Q \lambda)^{1 / 2} .
$$

We have

$$
S \ll \frac{M}{(Q R)^{1 / 2}}+\frac{M(Q \lambda)^{1 / 4}}{R^{1 / 2}}+\left(\frac{M S_{1}}{Q}\right)^{1 / 2}+\left(\frac{M S_{3}}{H Q R}\right)^{1 / 2}+M^{1 / 2} Q^{1 / 2} .
$$

(c) Now, we have to give a bound for $S_{3}$. We get a new variable by applying Weyl's shift on the variable $m$ :

$$
\begin{aligned}
S_{3} \ll \frac{1}{N} \sum_{m=Q+1}^{M-Q-N} \mid \sum_{r \neq 0} & \sum_{q \neq 0} \sum_{h} \sum_{n=1}^{N} b(r, q, h) \\
& \times e\left(\frac{h}{H} f(m+q+n)-\frac{h+r}{H} f(m+n)\right) \mid+R Q H N .
\end{aligned}
$$


By Taylor's formula, we can write

$$
\begin{aligned}
\frac{h}{H} f(m+q+n)-\frac{h+r}{H} & f(m+n) \\
= & -\frac{r}{H} f(m)+\frac{f^{\prime}(m)}{H}(h q-r n) \\
& +\frac{f^{\prime \prime}(m)}{2 H}\left(h q^{2}+2 h q n-r n^{2}\right)+\varphi_{m}(r, q, h, n) .
\end{aligned}
$$

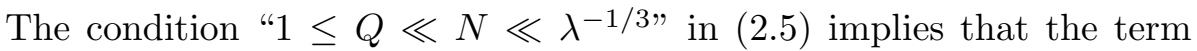
$\varphi_{m}(r, q, h, n)$ is small enough and can be removed by a four-dimensional summation by parts without cost as in Lemma 2 of [7]. Splitting the summation on $q$ according to its size, we finally get the inequality

$$
\begin{aligned}
& S_{3} \ll \frac{\log M}{N} \max _{1 \leq Q_{1}<Q} \sum_{m=Q+1}^{M-Q-N} \mid \sum_{r \neq 0} \sum_{Q_{1} \ll|q|<2 Q_{1}} \sum_{h=H+1}^{2 H} \sum_{n=1}^{N} c(r, q, h, n) \\
& \times e\left(-\frac{r}{H} f(m)+\frac{f^{\prime}(m)}{H}(h q-r n)+\frac{f^{\prime \prime}(m)}{2 H}\left(h q^{2}+2 h q n-r n^{2}\right)\right) \mid \\
& + \text { RQHN, }
\end{aligned}
$$

where the complex numbers $c(r, q, h, n)$ have modulus at most one.

(d) We conclude by applying Bombieri and Iwaniec's double large sieve to the above sum which is of the form

$$
\sum_{m} \sum_{r, q, h, n} a(m) c(r, q, h, n) e\left(\sum_{i=1}^{3} x_{i}(m) y_{i}(r, q, h, n)\right) .
$$

Thus, by Lemma 7.5 of [1], or Lemma 5.6.6 of [4], we get

$$
S_{3} \ll \frac{\log M}{N} \mathcal{B}^{1 / 2}\left(M \lambda R H Q^{2} N\right)^{1 / 2} \max _{1 \leq Q_{1} \ll Q} \mathcal{N}\left(R, Q_{1}, H, N ; \frac{1}{M \lambda Q_{1}^{2}}\right)^{1 / 2} .
$$

If we apply Lemma 1 and if we take (2.5) into account, we finally obtain

$$
S_{3} \ll_{\varepsilon} R Q^{3 / 2} H M^{1 / 2+\varepsilon} \lambda^{1 / 2} \mathcal{B}^{1 / 2}+R Q H N .
$$

We take back (2.10) into (2.8), and this gives (2.6).

REMARK. We have somewhat condensed the proof of Lemma 3. The reader interested in more details should refer to $\S 4$ of [7]. However, Lemma 3 is not exactly contained in [7].

As said above, an improvement of Lemma 2 is still possible with more hypotheses on $f$. But if we only suppose (1.1), Lemma 2 seems to be best possible. The following lemma follows at once from Lemmas 2 and 3. 
Lemma 4. Suppose that the hypotheses of Lemma 3 are satisfied and that

$$
\lambda H Q^{2} N \gg 1 \text {. }
$$

Then

$$
S \ll_{\varepsilon} M^{\varepsilon}\left(\frac{M}{(Q R)^{1 / 2}}+M(Q \lambda)^{1 / 4}+\left(\frac{M S_{1}}{Q}\right)^{1 / 2}+(M N)^{1 / 2}\right)
$$

3. Proof of Theorem 1. We split the proof of Theorem 1 into two steps. In the first one, we use Lemma 4 to get a slightly weaker form of Theorem 1. In the second one, we use the above result to bound $S_{1}$ in Lemma 4. This means that Lemma 4 is iterated once to prove Theorem 1.

3.1. An intermediate step

Lemma 5. Let $f:[1, M] \rightarrow \mathbb{R}$ be a $C^{3}$ function which satisfies (1.1). Define $S$ as in (1.7). Then

$$
S \ll_{\varepsilon} M^{\varepsilon}\left(\frac{M \lambda^{1 / 6}}{H^{1 / 9}}+M \lambda^{13 / 66}+M^{3 / 4}\right)+\lambda^{-1 / 3} .
$$

Proof. (a) We suppose first that $M<\lambda^{-2 / 3}$. Then Lemma 5 is not stronger than (1.3). Indeed, by (1.3), we have

$$
S \ll M \lambda^{1 / 6}+\lambda^{-1 / 3} \ll M^{3 / 4}+\lambda^{-1 / 3}
$$

and this is better than (3.1).

(b) We set $H_{0}=\lambda^{-3 / 11}$ and we suppose now that

$$
M \gg \lambda^{-2 / 3} \text { and } 10 \leq H \ll H_{0} .
$$

In Lemma 4, we fix the size of the parameters $R, Q, N$ :

$$
\begin{gathered}
R \asymp H^{2 / 3}, \quad Q \asymp \lambda^{-1 / 3} H^{-4 / 9}+(M \lambda)^{-1}, \\
N \asymp \lambda^{-1 / 3} H^{-1 / 9}+(M \lambda)^{-1} .
\end{gathered}
$$

For this choice of $R, Q, N$, it is easy to see that all conditions in (2.5) and (2.11) are satisfied, so that Lemma 4 may be applied:

$$
S \ll_{\varepsilon} \frac{M^{1+\varepsilon}}{(Q R)^{1 / 2}}+M^{1+\varepsilon}(Q \lambda)^{1 / 4}+(M N)^{1 / 2+\varepsilon}+\left(\frac{M^{1+\varepsilon} S_{1}}{Q}\right)^{1 / 2}
$$

and thus

$$
S \ll_{\varepsilon} \frac{M^{1+\varepsilon} \lambda^{1 / 6}}{H^{1 / 9}}+M^{3 / 4+\varepsilon}+\left(\frac{M^{1+\varepsilon} S_{1}}{Q}\right)^{1 / 2}
$$

with

$$
S_{1}=\frac{1}{R} \sum_{r=1}^{R}\left|\sum_{m=1}^{M} e\left(\frac{r}{H} f(m)\right)\right| \text {. }
$$


By (1.3), we get

$$
S_{1} \ll M\left(\frac{R \lambda}{H}\right)^{1 / 6}+\left(\frac{H}{R \lambda}\right)^{1 / 3} .
$$

We recall that $Q^{-1 / 2} \ll \min \left(\lambda^{1 / 6} H^{2 / 9},(M \lambda)^{1 / 2}\right)$, so that

$$
\left(\frac{M S_{1}}{Q}\right)^{1 / 2} \ll M \lambda^{1 / 4} H_{0}^{7 / 36}+M \lambda^{1 / 3} H_{0}^{1 / 18} \ll M \lambda^{13 / 66} .
$$

We take back this bound into (3.4) and we recover (3.1).

(c) We suppose now that

$$
M \gg \lambda^{-2 / 3} \text { and } H \gg H_{0} \text {. }
$$

We choose our parameters as follows:

$$
\begin{gathered}
R \asymp H / H_{0}^{1 / 3}, \quad Q \asymp \lambda^{-1 / 3} H_{0}^{-4 / 9}+(M \lambda)^{-1}, \\
N \asymp \lambda^{-1 / 3} H_{0}^{-1 / 9}+(M \lambda)^{-1} .
\end{gathered}
$$

Conditions (2.5) and (2.11) are again satisfied, and Lemma 4 yields

$$
S \ll_{\varepsilon} M^{1+\varepsilon} \lambda^{13 / 66}+M^{3 / 4+\varepsilon} .
$$

This completes the proof of Lemma 5.

3.2. Proof of Theorem 1. Let $f:[1, M] \rightarrow \mathbb{R}$ be a $C^{3}$ function which satisfies (1.1) and let $S$ be defined by (1.7). We have to prove that

$$
S \ll_{\varepsilon} \frac{M^{1+\varepsilon} \lambda^{1 / 6}}{H^{1 / 9}}+M^{3 / 4+\varepsilon}+M^{1+\varepsilon} \lambda^{1 / 5}+\lambda^{-1 / 3} .
$$

As in the proof of Lemma 5, we may suppose that $M \gg \lambda^{-2 / 3}$. We split the proof into two cases.

(a) We set $H_{1}=\lambda^{-3 / 10}$ and we assume that

$$
M \gg \lambda^{-2 / 3} \text { and } 10 \leq H \ll H_{1} \text {. }
$$

In order to apply Lemma 4, we introduce the parameters $R, Q, N$ and we fix their size as in (3.3). The conditions (2.5) and (2.11) are satisfied and we get (3.4) as in the previous proof.

The difference with the proof of Lemma 5 occurs here. Instead of (1.3), we use Lemma 5 to bound $S_{1}$ :

$$
\begin{aligned}
S_{1} & =\frac{1}{R} \sum_{r=1}^{R}\left|\sum_{m=1}^{M} e\left(\frac{r}{H} f(m)\right)\right| \\
& \ll \log M \max _{1 \leq R_{1} \leq R} \frac{R_{1}}{R} \cdot \frac{1}{R_{1}} \sum_{r=R_{1}+1}^{2 R_{1}}\left|\sum_{m=1}^{M} e\left(\frac{r}{R_{1}} \cdot \frac{R_{1}}{H} f(m)\right)\right|
\end{aligned}
$$




$$
\begin{aligned}
\ll_{\varepsilon} & \frac{R_{1}}{R} M^{1+\varepsilon} \frac{\left(R_{1} H^{-1} \lambda^{1 / 6}\right)}{R_{1}^{1 / 9}}+\frac{R_{1}}{R} M^{1+\varepsilon}\left(R_{1} H^{-1} \lambda\right)^{13 / 66} \\
& +M^{3 / 4+\varepsilon}+\frac{R_{1}}{R}\left(\frac{H}{\lambda R_{1}}\right)^{1 / 3} .
\end{aligned}
$$

In each term of the above formula, $R_{1}$ appears only with positive exponent so that we may take $R_{1}=R=H^{2 / 3}$ :

$$
S_{1} \ll_{\varepsilon} \frac{M^{1+\varepsilon} \lambda^{1 / 6}}{H^{7 / 54}}+\frac{M^{1+\varepsilon} \lambda^{13 / 66}}{H^{13 / 198}}+M^{3 / 4+\varepsilon}+H^{1 / 9} \lambda^{-1 / 3} .
$$

As we have chosen $Q \asymp \lambda^{-1 / 3} H^{-4 / 9}+(M \lambda)^{-1}$, we may write $Q^{-1 / 2} \ll$ $\lambda^{1 / 6} H^{2 / 9}$, or $Q^{-1 / 2} \ll\left(\lambda^{1 / 3} H^{4 / 9}\right)^{3 / 8}(M \lambda)^{1 / 8}=M^{1 / 8} \lambda^{1 / 4} H^{1 / 6}$, or $Q^{-1 / 2} \ll$ $(M \lambda)^{1 / 2}$, so that we have

$$
\begin{gathered}
\frac{M^{1 / 2} S_{1}^{1 / 2}}{Q^{1 / 2}} \ll_{\varepsilon} M^{1+\varepsilon} \lambda^{1 / 4} H^{17 / 108}+M^{1+\varepsilon} \lambda^{35 / 132} H^{25 / 132} \\
+M^{1+\varepsilon} \lambda^{1 / 4} H^{1 / 6}+M^{1+\varepsilon} \lambda^{1 / 3} H^{1 / 8} \\
\ll_{\varepsilon} M^{1+\varepsilon} \lambda^{1 / 4} H_{1}^{1 / 6} \ll_{\varepsilon} M^{1+\varepsilon} \lambda^{1 / 5} .
\end{gathered}
$$

We take back this bound into (3.4), and we recover (3.7) in this case.

(b) We now assume that

$$
M \gg \lambda^{-2 / 3} \text { and } H \gg H_{1} .
$$

The size of the parameters $R, Q$ and $N$ must be changed to

$$
\begin{gathered}
R \asymp H / H_{1}^{1 / 3}, \quad Q \asymp \lambda^{-1 / 3} H_{1}^{-4 / 9}+(M \lambda)^{-1}, \\
N \asymp \lambda^{-1 / 3} H_{1}^{-1 / 9}+(M \lambda)^{-1} .
\end{gathered}
$$

Conditions (2.5) and (2.11) are again satisfied and Lemma 4, together with the bound

$$
\frac{M^{1 / 2} S_{1}^{1 / 2}}{Q^{1 / 2}} \ll_{\varepsilon} M^{1+\varepsilon} \lambda^{1 / 5},
$$

yield

$$
S \ll_{\varepsilon} M^{3 / 4+\varepsilon}+M^{1+\varepsilon} \lambda^{1 / 5}
$$

from which we deduce (3.7). Theorem 1 is now proved.

\section{Application to lattice points problems}

4.1. Fractional parts and exponential sums. Let $g:[1, M] \rightarrow \mathbb{R}$ be any function. The sum

$$
\tau_{M}=\sum_{m=1}^{M} \psi(g(m))
$$


can be expressed in terms of mean values of exponential sums by means of the following classical lemma, which is a weaker form of Theorem A.6 of [1].

Lemma 6. Let $H$ be any positive integer. Then

$$
\tau_{M} \ll \frac{M}{H}+\sum_{h=1}^{H} \frac{1}{h}\left|\sum_{m=1}^{M} e(h g(m))\right| .
$$

4.2. Proof of Theorem 2. We suppose furthermore that $g$ is a $C^{3}$ function which satisfies van der Corput's hypothesis

$$
g^{\prime \prime \prime}(x) \asymp \lambda, \quad 1 \leq x \leq M,
$$

and we have to prove that

$$
\tau_{M} \ll_{\varepsilon} M^{1+\varepsilon} \lambda^{3 / 19}+M^{3 / 4+\varepsilon}+\lambda^{-1 / 3} .
$$

Let $H$ be a positive integer. From Lemma 6 , we deduce that

$$
\tau_{M} \ll \frac{M}{H}+\sum_{R} \frac{1}{R} \sum_{h=R+1}^{2 R}\left|\sum_{m=1}^{M} e(h g(m))\right|,
$$

where $R$ runs over all possible values which are powers of 2 smaller than $H$. We are now in a position to apply Theorem 1 . This yields

$$
\begin{aligned}
\tau_{M} & \ll \varepsilon \frac{M}{H}+\sum_{R}\left(M^{1+\varepsilon} R^{1 / 18} \lambda^{1 / 6}+M^{1+\varepsilon} R^{1 / 5} \lambda^{1 / 5}+M^{3 / 4+\varepsilon}+\frac{\lambda^{-1 / 3}}{R^{1 / 3}}\right) \\
& \ll_{\varepsilon} \frac{M}{H}+M^{1+\varepsilon} H^{1 / 18} \lambda^{1 / 6}+M^{1+\varepsilon} H^{1 / 5} \lambda^{1 / 5}+M^{3 / 4+\varepsilon}+\lambda^{-1 / 3} .
\end{aligned}
$$

The choice of $H$ is determined by Srinivasan's Lemma (cf. [1, Lemma 2.4]), that is, $H \asymp \lambda^{-3 / 19}$, and this implies (4.3).

4.3. Integer points close to a curve. To illustrate our Theorems 1 and 2 in terms of lattice points, we choose the problem of integer points close to a curve. Let $g:[1, M] \rightarrow \mathbb{R}$ be any function, and let $\delta(0<\delta<1 / 2)$ be a real number. We set

$$
\mathcal{R}(g, \delta)=\#\{m \in\{1, \ldots, M\} \mid\|g(m)\| \leq \delta\} .
$$

Two problems can be considered. The first one consists in finding an upper bound for $\mathcal{R}(g, \delta)$, while the second consists in finding the asymptotic behaviour of $\mathcal{R}(g, \delta)$, that is, in writing $\mathcal{R}(g, \delta)=2 M \delta+E$, where $E$ is an error term.

4.3.1. An upper bound for $\mathcal{R}(g, \delta)$. The problem reduces to exponential sums by means of the following lemma. 
Lemma 7. Let $H$ be a positive integer, $H \ll \delta^{-1}$. Then

$$
\mathcal{R}(g, \delta) \ll \frac{M}{H}+\frac{1}{H} \sum_{h=1}^{H}\left|\sum_{m=1}^{M} e(h g(m))\right| .
$$

Proof. This is a weaker form of Lemma 5.3.2 of [4].

Under the assumption

$$
g^{\prime \prime \prime}(x) \asymp \lambda \quad \text { for } 1 \leq x \leq M,
$$

we deduce from (1.3) and Lemma 7 that

$$
\mathcal{R}(g, \delta) \ll M \delta+M \lambda^{1 / 7}+M^{2 / 3}+\left(\frac{\delta}{\lambda}\right)^{1 / 3} .
$$

For small values of $\delta$, the method of divided differences yields the better bound (cf. [5])

$$
\mathcal{R}(g, \delta) \ll M \delta^{1 / 3}+M \lambda^{1 / 6}+\left(\frac{\delta}{\lambda}\right)^{1 / 3} .
$$

From Lemma 7 and Theorem 1, we deduce at once the following bound.

Corollary 1. If we assume (4.5), then

$$
\mathcal{R}(g, \delta) \ll_{\varepsilon} M \delta+M^{1+\varepsilon} \lambda^{3 / 19}+M^{3 / 4+\varepsilon}+\left(\frac{\delta}{\lambda}\right)^{1 / 3} .
$$

Proof. By Lemma 7, we reduce the problem to the estimate of exponential sums which depend on a parameter $h$. By Theorem 1, we arrive at the bound $\mathcal{R}(g, \delta) \ll_{\varepsilon} \frac{M}{H}+M^{1+\varepsilon} \lambda^{1 / 6} H^{1 / 18}+M^{1+\varepsilon} \lambda^{1 / 5} H^{1 / 5}+M^{3 / 4+\varepsilon}+(H \lambda)^{-1 / 3}$, with the restriction $1 \leq H \ll \delta^{-1}$.

We optimize this bound depending on the parameter $H$ as in Srinivasan's Lemma (cf. [1, Lemma 2.4]). We get

$$
\mathcal{R}(g, \delta) \ll_{\varepsilon} M \delta+M^{1+\varepsilon} \lambda^{3 / 19}+M^{3 / 4+\varepsilon}+\left(\frac{\delta}{\lambda}\right)^{1 / 3}+M^{6 / 7+\varepsilon} \lambda^{2 / 21},
$$

because the remaining terms given by Srinivasan's Lemma are $M^{1+\varepsilon} \lambda^{1 / 6}$ and $M^{5 / 8+\varepsilon}$ and can be obviously removed. But the term $M^{6 / 7+\varepsilon} \lambda^{2 / 21}$ can also be removed; indeed, we have

$$
M^{6 / 7} \lambda^{2 / 21} \leq M^{6 / 7} \lambda^{9 / 133}=\left(M^{3 / 4}\right)^{4 / 7}\left(M \lambda^{3 / 19}\right)^{3 / 7} \leq M^{3 / 4}+M \lambda^{3 / 19} .
$$

The proof of Corollary 1 is complete. 
4.3.2. Asymptotic behaviour of $\mathcal{R}(g, \delta)$. In the next lemma, we reduce the problem of the asymptotic behaviour of $\mathcal{R}(g, \delta)$ to estimating the sum $\sum_{m=1}^{M} \psi(g(m) \pm \delta)$.

Lemma 8. Let $g:[1, M] \rightarrow \mathbb{R}$ be any function. Then for any $\delta \in[0,1 / 2[$, we have

$$
\mathcal{R}(g, \delta)=2 M \delta+E
$$

with

$$
|E| \leq 2 \max _{-1 / 2 \leq \vartheta \leq 1 / 2}\left|\sum_{m=1}^{M} \psi(g(m)+\vartheta)\right| .
$$

Proof. We set

$$
\mathcal{R}_{\vartheta}=\sum_{m=1}^{M}([g(m)+\delta]-[g(m)-\vartheta]) .
$$

It is easy to see that

$$
\mathcal{R}(g, \delta)=\lim _{\vartheta \rightarrow \delta+0} \mathcal{R}_{\vartheta}
$$

We have

$$
\begin{aligned}
\mathcal{R}_{\vartheta} & =\sum_{m=1}^{M}((g(m)+\delta-\{g(m)+\delta\})-(g(m)-\vartheta-\{g(m)-\vartheta\})) \\
& =\sum \sum_{m=1}^{M}(\delta+\vartheta-\psi(g(m)+\delta)+\psi(g(m)-\vartheta)) \\
& =\sum M(\delta+\vartheta)+E_{1}+E_{2}
\end{aligned}
$$

with

$$
\left|E_{i}\right| \leq \max _{-1 / 2 \leq t \leq 1 / 2}\left|\sum_{m=1}^{M} \psi(g(m)+t)\right|,
$$

provided that $\vartheta<1 / 2$. By letting $\vartheta$ tend to $\delta+0$, we get (4.8).

The following corollary can be deduced at once from Lemma 8 and Theorem 2 . have

Corollary 2. Assume that (4.5) holds. Then, for any $\delta \in[0,1 / 2[$, we

$$
\mathcal{R}(g, \delta)=2 M \delta+O_{\varepsilon}\left(M^{1+\varepsilon} \lambda^{3 / 19}+M^{3 / 4+\varepsilon}\right)+O\left(\lambda^{-1 / 3}\right) .
$$

\section{References}

[1] S. W. Graham and G. Kolesnik, Van der Corput's Method for Exponential Sums, London Math. Soc. Lecture Notes Ser. 126, Cambridge Univ. Press, 1991. 
[2] G. Grekos, Sur le nombre de points entiers d'une courbe convexe, Bull. Sci. Math. (2) 112 (1988), 235-254.

[3] S. A. Gritsenko, Estimates of trigonometric sums by the third derivative, Math. Notes 60 (1996), 283-287.

[4] M. N. Huxley, Area, Lattice Points and Exponential Sums, Clarendon Press, Oxford, 1996.

[5] M. N. Huxley et P. Sargos, Points entiers au voisinage d'une courbe plane de classe $C^{n}$, Acta Arith. 69 (1995), 359-366.

[6] E. Krätzel, Lattice Points, Deutscher Verlag Wiss., Berlin, 1988.

[7] O. Robert and P. Sargos, A fourth derivative test for exponential sums, Compositio Math. 130 (2002), 275-292.

[8] P. Sargos, Points entiers au voisinage d'une courbe, sommes trigonométriques courtes et paires d'exposants, Proc. London. Math. Soc. (3) 70 (1995), 285-312.

[9] —, La méthode de Bombieri et Iwaniec pour les sommes d'exponentielles, in: Journées Elie Cartan 1998 et 1999, Publ. Inst. Elie Cartan no. 16, 118-140.

Institut Elie Cartan

Université Henri Poincaré - Nancy I

BP 239

54506 Vandœuvre-lès-Nancy Cedex, France

E-mail: Robert@iecn.u-nancy.fr

Sargos@iecn.u-nancy.fr

Received on 5.12.2000

and in revised form on 25.3.2002 